\title{
Editorial
}

\section{Just Another Cost Increasing Exercise (JACIE)?}

\author{
Bone Marrow Transplantation (2004) 34, 835-838. \\ doi:10.1038/sj.bmt.1704651
}

The year 2004 will see the real start of implementation of a pan-European series of standards for the practice of stem cell transplantation. In all, 25 teams from Switzerland, Austria, France, Belgium, The Netherlands and the United Kingdom will be inspected for the purposes of accreditation by the Joint Accreditation Committee of International Society of Cellular Therapy, Europe (ISCT, formerly ISHAGE) and the European Group for Blood and Marrow Transplantation (EBMT) (JACIE). The goal is to have the majority of European transplant teams inspected by 2008 .

JACIE was created in 1997 and approved the first set of standards for haemopoietic progenitor cell collection, processing and transplantation ${ }^{1}$ in 1998. These guidelines were heavily based on those published the previous year in the USA, ie, the standards of the Foundation for the Accreditation of Haematopoietic Cell Therapy (FACT). Throughout the process the boards of JACIE and FACT have worked closely together to harmonise the standards. The second editions of both sets of standards were produced in 2002 (FACT) and 2003 (JACIE). The current structure of JACIE is shown in Table 1 and the Board have made particular efforts to ensure wide consultation with the 13 European countries being represented on the Executive Committee.

The goal of the accreditation processes in the USA and Europe is to improve the quality of patient care. The scope of the standards is broad. They cover both donors and recipients and all phases of collection, processing, storage and administration of haemopoietic progenitor cells. The standards are set for processes rather than products and are the basis of a voluntary accreditation programme. For the first time, the standards for education and training, the development of a wider-ranging quality management system (QMS) and the reporting of all clinical outcomes are set out in detail. It is worth pointing out that neither FACT nor JACIE intrude on physician decisions with respect to the appropriateness of the transplant procedure; they instead address the way in which the procedure is carried out, that is, once the transplant is planned it should be performed according to these standards.

The original intent of JACIE had been to establish and agree on a set of standards based on consensus of experts in the field, make these available to individual teams and nations and oversee a process where the details of inspection and accreditation were devolved to the National Transplant Societies. Progress was somewhat slower than originally intended and it rapidly became clear that there were specific reasons why adoption of the process was delayed. Most National Societies lacked the infrastructure to implement a system of training (for applicant centres and for inspectors), inspection and accreditation. Most centres had little real understanding of the amount of work required to reach the 'inspection ready' point, and to be honest, many physicians doubted the need for such an elaborate and 'paper heavy' system. Over all these doubts and objections, the most important issue was a lack of financial support from any source to implement the process. Most European countries with their different approaches to health care were reluctant to release additional funds for voluntary - as opposed to legal and obligatory - requirements.

By the end of 2002, when only four centres in Spain had achieved JACIE accreditation, the process was re-evaluated. After discussion with the National Societies, the central office of JACIE decided to take back some of the previously devolved responsibilities. This was facilitated by the award of a grant from the European Union Public Health Programme, matched equally by monies from EBMT and the National Societies. This permitted the establishment of a permanent JACIE office in Barcelona, with staff specifically dedicated to the project, additional support for education and training of both inspectors and transplant teams, supervision of the documentation, devel-

Table 1 JACIE executive board

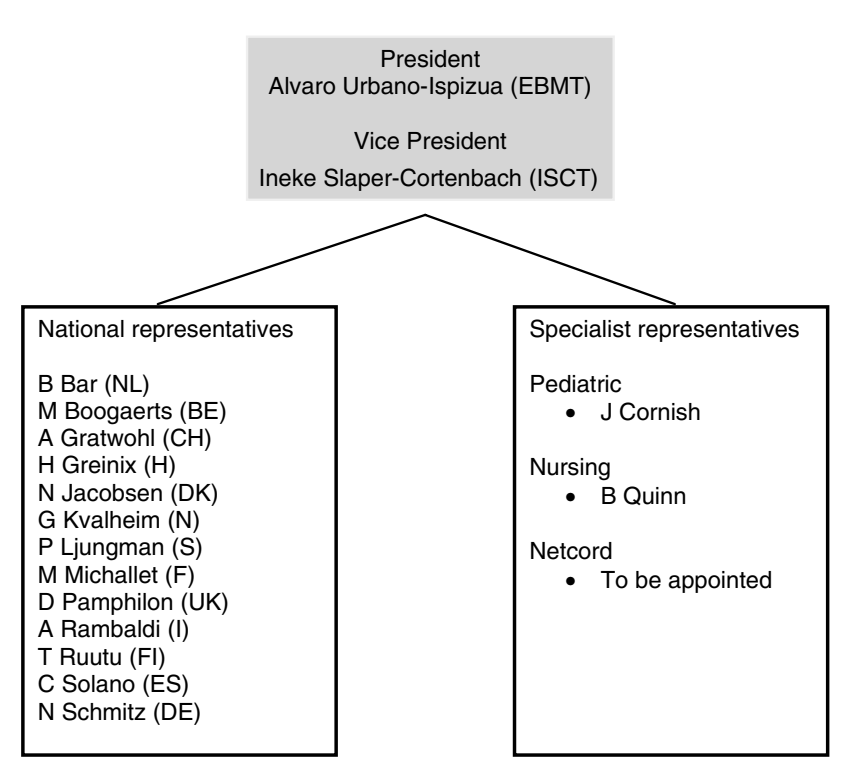


opment of an appeal process, establishment of a web-site and on-line registration and document submission. The milestone at the end of the first year was to have inspected, and hopefully accredited, 25 individual teams. Nations with the capacity to take on training and inspections will do so in close collaboration with the Barcelona office, and those without such an infrastructure will be inspected directly by JACIE. Accreditation will be awarded from the Barcelona office and not by individual National Societies. To date, the process is entirely on schedule. Applications for continuing funding for this central process are currently being reviewed.

So, excellent progress in the past 12 months, but is it all worth it? Where is the evidence that any part of the process will improve patient care, drive up quality standards or change the outcome of any single transplant procedure? What is the cost? And how will this system integrate with the forthcoming EU directive on tissue banking?

In this issue of BMT, Zahnd and co-workers describe the cost involved in implementing JACIE standards for a moderately sized transplant unit at a University Hospital in Switzerland, performing 40 autografts per annum. ${ }^{2}$ The analysis was restricted to costing the time spent by personnel in making the unit ready for inspection. The number of hours involved was 945.5 and the total cost was calculated to be 150000 Euro. It is difficult to know exactly how these costs will compare with those that will be incurred by other centres because it is difficult to define a 'typical' centre. Of the 586 transplant units which reported their activity levels to the annual EBMT survey in 2002, ${ }^{3}$ $230(39 \%)$ perform autologous transplants only. As this team carries out only autologous transplants, they will have spared themselves the additional effort of addressing the needs for extra facilities, training, protocols for the complications of allografting, required for the practice of allogeneic transplantation, third-party agreements for receipt of unrelated products, etc. However, this particular unit treats both paediatric and adult patients, which is relatively unusual for autologous centres. This has undoubtedly introduced some added complexity and cost with respect to meeting standards for both patient populations.

If we are to extrapolate this information to gain some idea of the total costs for accreditation across Europe, it is necessary to have some understanding of the provision of transplants (Figure 1). In all, $50 \%$ of all transplants reported to the annual EBMT survey are carried out by $20 \%$ of the teams, that is, the largest teams, most of whom are performing both autologous and allogeneic transplantation. A total of $50 \%$ of the teams, ie, the smallest teams, carry out $17 \%$ of the total number of transplants. Although complete data are not available, it is quite likely that many of these teams will share laboratory facilities with larger teams or obtain their stem cell products from sources such as their National Blood Authority. They might therefore be spared the accreditation costs for the laboratory. It would be reasonable to assume that Dr Zahnd's unit is typical of a small to moderately sized unit in terms of transplant numbers and complexity of processing. Such units comprise $30 \%$ of the 586 centres. If we make the cost calculations based on some relatively simple premises, that is to say, that the costs of the largest centres will be at least one-third

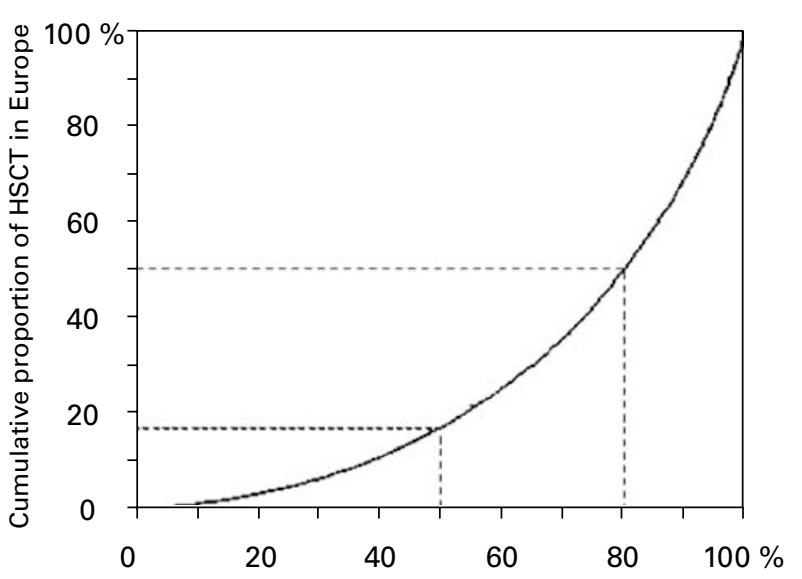

Cumulative proportion of teams performing HSCT in Europe

Figure 1 Graphical representation of the numbers of transplants performed in Europe by teams reporting to the annual EBMT activity survey.

more and that the smaller centres are likely only to save on their laboratory costs, which comprise about one-third of the work-load, then the total cost for all the European centres (for the development of their QM systems) is a staggering 79 million Euro.

How do these costs accumulate? The process took a total of 14 months, and at the time of submission of the manuscript, the results of the inspection were not known. The analysis deliberately excluded the costs of the inspection itself and the costs of maintaining and developing a QMS. These will be the subject of a further analysis after accreditation. In addition, the laboratory facilities were already compliant with the European norm, EN ISO/IEC 17025, which is more stringent than JACIE, and so there was no need to invest in the laboratory infrastructure.

The authors freely acknowledged that one potential impact of the European Union directive on tissue banking will be to raise current standards within hospital units to those of good manufacturing practice (GMP), which in turn will increase costs. A recent survey of current status of UK facilities for stem cell processing and storage requested information on the estimated costs of raising the standards to GMP (British Society of Blood and Marrow Transplant, personal communication). Depending on the number of products processed, the complexity of the procedures, the current status of the laboratory fabric and the ability - or not - to use pre-existing space, estimates varied justifiably from 100000 to 3000000 Euro. This survey also specifically excluded the needs of increasing and maintaining adequate staffing levels and the introduction of IT systems for equipment and product databases. The level of increased investment needed came as a surprise to UK Department of Health officials and no additional monies had been set aside.

The huge cost involved has caused many within the field of transplantation to question the need for - or the advisability of - the standards. Critics of the system make a number of points. There is a massive investment of time by individuals whose attentions might be better directed to patient care, clinical trials and/or basic science. The imposition of rigid protocols presupposes that one way is 
the best way and stifles innovation. The use of standard operating procedures (SOPs) assumes that all patients behave identically and underestimates the value of experience and clinical instinct. QMSs require validation, but this is an area in which the rules for validation have not always been written. For instance, we know that cell dose may be very important in clinical outcome, but one can hardly discard an unrelated stem cell product that does not meet predetermined standards.

The proponents of accreditation often draw the analogy with the airline industry. There, a quality assurance system stipulates written procedures for all activities, strict adherence to such SOPs, recording of errors, review, audit and continuing training. The risk of human error must be and has been minimised. Why can't the same principle be applied to clinical medicine? Well it can, but it requires some uncomfortable decisions on the part of our society. The extreme consequences of error on board a jumbo jet include the deaths of all staff and passengers, loss of valuable cargo, subsequent insurance claims, insolvency of the airline company and the loss of jobs directly or indirectly associated with that company. In other words, even setting aside the emotional consequences, the financial repercussions are huge. Small wonder that the industry is prepared to invest so heavily on.

McKenna et $a l^{4}$ have recently described the establishment of a system to report and evaluate errors and adverse reactions within their cellular therapy laboratory. Their system does not collect data on similar events in the clinical part of their programme. Their laboratory is perhaps not typical of stem cell processing units as more than 1700 of the 2000 products were derived from umbilical cord blood. In the first year, 335 events affecting 385 products (19\%) were reported, recorded, investigated, discussed and acted upon. The events were assessed for their impact on both the product and the patient. Only one event was given the highest grade of severity with respect to the patient, ie, a possible impact on the clinical outcome of this individual could not be excluded. So, the extreme consequence of an error in a transplant unit is the loss of a single life of an individual who, without the transplant, stood a sizeable risk of death from their disease. It could be argued that although the personal tragedy would be great, the financial impact would be relatively minor, despite a risk of possible litigation. Hence, the issue for society. How much money should be spent in health-care provision to prevent the loss of a single life? In other words, what is the worth of the life of a leukaemia patient? And, if the provision of a QMS by a cash-strapped health service reduces the total number of transplants that can be performed, where is the benefit to society?

We live in a world increasingly concerned with personal risk, and recent years have seen a proliferation of health and safety activities. However, spending increasing amounts of money on reducing the risk of treatment to a minimum may be simply ignoring the fact that simply having a haematological disease is in itself a danger to the sufferer. Perhaps, the regulators should remember that sometimes even dangerous treatment might be better than no treatment at all. And anyway, who regulates the regulators?
The alternative (and unassailable) argument relates to the fact that an unrelated stem cell transplant is one of the most expensive, if not the most expensive, elective medical procedures with the least chance of success. At the risk of complaints from numerous single centres with excellent results, let us assume a 50\% transplant-related mortality. Is there another procedure in health care with a similar costbenefit ratio? Isn't it therefore the responsibility of the transplant team to minimise every possible risk? Is it not also reasonable to give the patient a fighting chance of success, so that, when they submit themselves to transplant, irrespective of the geographical location of the unit, it is carried out in accordance with the best possible standards of care? Health-care providers very reasonably ask us to do this and therefore they will have to find the money to support such initiatives.

Improvements in the outcome of patients with haematological disorders have come from painstaking attention to detail which in turn has led to better selection of patients, improved supportive care and the rational introduction of novel agents. JACIE accreditation is part of this process and most transplant teams are performing to the standards of JACIE already. All that we are being asked to do is to document this. Furthermore, if we, as a group traditionally associated with cutting edge medicine, wish to continue to develop innovative methods of managing haematological diseases, then we must be accountable for our actions. We cannot begin to question the worth of an individual life, otherwise our daily work becomes meaningless.

Although Zahnd et al rightly draw attention to the costs of JACIE, they also freely admit some of the benefits in working their way through the process, and many of their positive findings have been reiterated by teams achieving FACT accreditation in the USA. The development of clear written protocols has allowed the delineation of responsibilities and provided invaluable tools for information and training. In addition, recording of the outcome of the procedures permits comparison with the expected outcome and facilitates early recognition and correction of errors. Other teams have commented on the positive effect that the involvement of all the staff of the unit has had on team working and commitment. Better working relationships can only have a positive benefit for patients.

For all these reasons, JACIE is essential. In fact, JACIE should be welcomed with open arms by all transplant units. It is an outstanding example of a situation in which the professionals have successfully instituted a transparent system of self-regulation, thereby pre-empting the imposition of a set of standards by faceless bureaucrats. Far be it for me to mention the EU directive on clinical trials! JACIE's structure and transparency are its strengths. We are regulating ourselves in an accountable manner. All transplant staff have an opportunity to participate in the process, highlight problems, make suggestions and determine its success by their willingness to subject their units to inspection. Widespread uptake over the next few years will render the development of any other process extremely difficult. Our peers have stood up to be counted and should be congratulated and fully supported. 
J Apperley

Department of Haematology,

Faculty of Medicine,

Imperial College London,

Hammersmith Hospital Campus,

Du Cane Road,

London W12 ONN, UK

\section{References}

1 Joint Accreditation Committee of the International Society for Cellular Therapy Europe and the European Group for Blood and Marrow Transplantation (JACIE). JACIE standards for hematopoietic progenitor cell collection, processing and transplantation. JACIE 1998.
2 Zahnd D, Leibundgut $\mathrm{K}$, Zenhausern $\mathrm{R}$ et al. Implementation of the JACIE standards for a haematopoietic progenitor cell transplantation program: a cost-analysis. Bone Marrow Transplant 2004; 34: 847-853.

3 Gratwohl A, Baldomero $\mathrm{H}$, Passweg $\mathbf{J}$ et al. Accreditation Committee of the European Group for Blood and Marrow Transplantation (EBMT); Working Parties Acute (ALWP) Chronic Leukemias (CLWP); Lymphoma Working Party. Hematopoietic stem cell transplantation for hematological malignancies in Europe. Leukemia 2003; 17: 941-959.

4 McKenna D, Kadidlo D, Dumstad D, McCullough J. Development and operation of a quality assurance system for deviations from standard operating procedures in a clinical cell therapy laboratory. Cytotherapy 2003; 5: 314-322. 